\title{
PARTICULARITIES SPECIFIC TO THE TRAINING OF GYMNASTS WITH DOWN SYNDROME FOR HIGH-LEVEL COMPETITIONS
}

\author{
Gabriel POPESCU ${ }^{1 *}$, Silvia STROESCU², \\ ${ }^{1}$ National University of Physical Education and Sport, Faculty of Physical Education and Sport, Bucharest, Romania \\ *Corresponding author: stroescusilvia@yahoo.com
}

DOI: $10.35189 /$ iphm.icpesk.2019.29

\begin{abstract}
The research aims to analyse the training process, the competitive performance and the results of the Romanian Gymnastics Team made up of 8 athletes ( 2 girls - level I, 2 girls - level II, 2 boys - level I, 2 boys - level II), who participated in the 2019 Special Olympics World Games held in Abu Dhabi (United Arab Emirates). Concise reference syntheses were produced regarding the specific training of gymnasts with Down syndrome, the dialectical relationship between training and performance, the individual behaviour of athletes in training and competition, the methodology and technology of their preparation for high-level competitions. Several parameters were studied: volume, number of hours/training; total number of training hours; number of full exercises/training; number of full exercises/training/ apparatus; total number of training sessions with specific programmes for physical support; progress of execution represented by individual and group charts; weekly learning curve; individual learning curve; strengthening, improving learning for boys/ girls; competitive performances, rankings and medals. The comparative data between boys and girls - from beginners to advanced, the volume-quality ratio, the values of the individual progress achieved by the 8 gymnasts (by level and gender) for the 30 studied parameters become benchmarks in the preparation of gymnasts with Down syndrome for top competitions, levels I and II, boys and girls.
\end{abstract}

Keywords: high-level gymnastics, gymnasts with Down syndrome, training, 2019 World Games results.

\section{Introduction}

For many years, our concern to train people with disabilities and especially people with Down syndrome DS) in performance artistic gymnastics - Special Olympics has been a top priority and a moral and ethical responsibility. The previous 5 articles on the same theme published internationally over the years speak for themselves.

Kerstiens and Green (2015) state that, although less well-understood, there is potential for improved motor learning in individuals with DS as a result of exercise participation. According to Popescu, Dina, Stroescu and Dina (2013), there have been constant attempts to demonstrate the potential of these special people to approach normality and even performance in motor, psychomotor, affective, mental and behavioural control in a sport discipline close to the art.

At the Abu Dhabi 2019 Special Olympics World Summer Games (WSG), where we participated with 3 gymnasts (one did not travel for special reasons) and 4 gymnasts with Down syndrome, at levels I and II, we won 30 medals: 12 gold, 9 silver and 11 bronze (SO Romania, 2019). They are added to the 9 medals won by the Romanian gymnasts, under the same guidance and coordination, at the previous edition of the Los Angeles 2015 Special Olympics World Summer Games.

The 2019 Special Olympics World Games, held between 14 and 21 March in Abu Dhabi, United Arab Emirates, were attended by athletes, coaches, volunteers and spectators from 190 countries, being considered the largest humanitarian sports event of the year (Monsen, 2019).

The Abu Dhabi 2019 Special Olympics World Games will be an unprecedented display of spirit, joy, courage and skill. In these Olympic Games, which take place every four year, beyond the achieved results, we had the opportunity to promote the Romanian gymnastics school and demonstrate the importance of creative, scientific, judicious training adapted to gymnasts with special needs.

The athletes' performance in the competition, the technical line presented and the obtained results justify the "key" reference elements as a model for the various highlights of these excellent athletes.

The training of gymnasts with Down syndrome is not easy at all, because they have to cope with multiple difficulties and many objective or subjective limiting factors (relating to design, preparation time, gymnasts' weight and heterogeneous experience, diet, socio-organizational conditions, ability of understanding, representation, learning, level of control and self-control, etc.). According to Ayers and Sariscsany (2011), it includes five components: cardiorespiratory endurance, muscle strength, muscle endurance, flexibility and body composition. 
In order to identify the training specifics for both men's but especially women's gymnasts, we conducted this study on the training of male and female gymnasts for the Abu Dhabi 2019 Special Olympics Summer Olympics.

\section{Hypothesis}

This scientific approach has started from the following hypothesis: in an original project for the preparation of male and female artistic gymnastics teams (Special Olympics Romania) to participate in the Abu Dhabi 2019 World Summer Games, the monitoring, observation, analysis, comparison and experiment can help identify, objectify and confirm the strategic and operational specifics of the training process, as well as qualitative and quantitative reference elements that can guide it.

\section{Purpose of the study}

It is in full harmony with the proposed goal of providing specialists from our country, Europe or around the world with data and concrete elements, objective reference points on the training of gymnasts with disabilities for high-level gymnastics competitions.

\section{Material and Methods}

\section{Experiment data: Duration, time, place, experiment group, organization, operational strategies}

The special training programme of the Romanian Artistic Gymnastics Team for the Abu Dhabi 2019 Special Olympics World Summer Games started on 15 September 2018 and lasted until 15 March 2019. For special reasons, it was divided into three periods: two training periods from 15 September to 15 December and from 15 January to 7 March, separated by the winter holidays (16 December 2018 - 14 January 2019), and a competitive period, from 12 to 15 March, when divisions and finals were held on World Series apparatus.

All the training sessions and preparatory competitions took place in the gym of the National University of Physical Education and Sport in Bucharest. Five to six weekly workouts of 2-2.30 hours were performed.

There were 8 gymnasts in this programme: 4 boys: 2 at level I, 2 at level II; 4 girls: 2 at level I, 2 at level II.

Each athlete benefited from a structured and individually tailored programme according to their level, experience and rate of progress. Putting it into practice was difficult and laborious, because of the very short time and multiple problems. Among other things, one of the level-I female gymnasts was a beginner on September 15, and the other 2 female gymnasts were level II and therefore had just started to learn the new exercises required for this level. This is why all 160 algorithmic programmes for the learning and improvement of elements were individualised, adjusted and adapted (see the item Levels I and II - Girls - Boys).

Starting from the constraints derived from both the requirements of sport performance and the conditional relationships between the training components, the operational objectives pursued were aimed at:

1. Achieving and maintaining the optimal weight for training and competition;

2. Achieving optimal parameters for specific physical training: strength, joint elasticity, balance, orientation in space and time;

3. Increasing learning ability, consolidating, refining and maintaining the level of performance;

4. Obtaining safety and constancy in execution;

5. Memorising the number of iterations of certain repetitive structures from various exercises (swings, change of grips, etc.);

6. Increasing concentration ability;

7. Overcoming fears (beams, uneven bars, parallel bars);

8. Increasing the ability to maintain elements of strength or balance;

9. Obtaining maximum range of motion for certain directions;

10. Raising awareness of the importance of capitalising on one's will, desire to work properly, enthusiasm and positive thinking;

11. Capitalising on psychomotor training;

12. Facilitating the formation of representations;

13. Having the "sense" of the apparatus, positions and basic movements.

According to Damian, Popa and Popa (2015), all of these goals can be identified as the first reference elements specific to the competition for people with Down syndrome. As many of these need to be solved by repetition while respecting the dialectical volume-quality ratio, other quantitative and qualitative reference elements can be 
added, which directly determines the monitoring, objectification and adjustment of gymnast training for the competition.

A. Quantitative parameters

1. Number of months of training

2. Number of weeks of training

3. Number of weeks. Rest/Holiday

4. Number of exercises performed

5. Number of hours/workout

6. Total number of training hours

7. Number of full routines/training

8. Number of full routines/training/apparatus

a. Levels I and II Girls: vault, uneven bars, floor, beam

b. Levels I and II Boys: floor, pommel horse, rings, vault, parallel bars, horizontal bar

9. Total number of full routines/apparatus

a. Levels I and II Girls: vault, uneven bars, floor, beam

b. Levels I and II Boys: floor, pommel horse, rings, vault, parallel bars, horizontal bar

10. Total number of training sessions with specific physical training programmes

11. Number of verification competitions/modelling competitions

12. Number of elements and parts of elements/apparatus/level/girls/boys

13. Total number of learning and improvement elements and parts of elements/apparatus/level/girls/boys

B. Qualitative parameters

14. Progress in the execution of elements/apparatus/level/boys/girls

15. Progress in the full routine/apparatus/level/boys/girls

16. Result progress in the all-around competition

17. Results obtained in the qualifying and finals competition

In order to objectify these parameters, the limiting indicators of progress in general and individual learning will be analysed, namely:

- excessive weight

- injury

- palm tearing

- inefficient ratio of the training components:

- $\quad$ specific physical training - technical training

- technical training - psychological training

- weekly learning curve that shows significant losses between the end and the beginning of the week

Comparative data of the training process (beginner and advanced girls and boys, or levels of training), as well as competitive behaviour (emotional management, safety, mobilisation, concentration) were also analysed.

\section{Results}

In the following examples, all quantitative assessments are given in terms of number and quality.

Comparative elements between male and female gymnasts

Through the comparative analysis of the training and the results obtained by male and female gymnasts (Tables 11 and 12), we identify:

- the importance of preserving and capitalising on the safety weight, optimal weight throughout the training, with a direct impact on the quality of the training and the competitive performance (Tables 1-5);

- the importance of determination and the desire to win (see results in the competition);

- the importance of the ability to control emotions and the positive management of emotional states (see results in the competition);

- the importance of motor skills and competition experience (see result difference between beginner and advanced gymnasts - Tables 11 and 12). 
An objective comparative analysis showed that all male gymnasts had been trained within the bodyweight limits imposed by safety and the progress of learning compared to female gymnasts, who reached the optimum chart only in the competition month.

In this regard, M.M. - level I had the greatest advantage: being strong, well-trained and $10 \mathrm{~kg}$ less, he was able to confirm his value by taking 3 gold and 2 silver medals.

Table 1. Weight changes in female gymnasts

\begin{tabular}{lcccccc}
\hline \multicolumn{1}{r}{ Year/ } & \multicolumn{3}{c}{2018} & & & 2019 \\
\cline { 2 - 7 } \multicolumn{1}{c}{ Month } & X & XI & XII & I & II & III \\
\hline M.M. & 73 & & 68 & 66 & 64 & $\mathbf{6 2 . 5}$ \\
M.B. & 48 & & 46 & & & $\mathbf{4 5 . 5}$ \\
I.A.M. & 52 & & 51 & & & $\mathbf{4 6}$ \\
I.D. & 48 & 47 & 46.5 & 46.5 & 46.5 & $\mathbf{4 5 . 5}$ \\
\hline
\end{tabular}

Table 2. Initial and final tests for the body mass index of female gymnasts

\begin{tabular}{lcc}
\hline \multicolumn{3}{c}{ Body mass index - Female gymnasts } \\
\multicolumn{1}{c}{ Name } & Initial test (IT) & Final test (FT) \\
\hline M.M. & 28.1 & 24.2 \\
M.B. & 23.8 & 21.9 \\
I.A.M. & 24.8 & 21.6 \\
I.D. & 23.4 & 22.8 \\
\hline
\end{tabular}

Table 3. Weight changes in male gymnasts

\begin{tabular}{lccccccc}
\hline Year/ & \multicolumn{3}{c}{2018} & & \multicolumn{3}{c}{2019} \\
\cline { 2 - 7 } Month & $\mathrm{X}$ & $\mathrm{XI}$ & $\mathrm{XII}$ & $\mathrm{I}$ & $\mathrm{II}$ & $\mathrm{III}$ \\
\hline S.I. & 56.5 & 56.5 & 56.5 & 56.5 & 56.5 & 56 \\
P.I. & 58.5 & 58 & 58 & 57 & 57 & $\mathbf{5 6}$ \\
M.A. & 55.5 & 56 & 55 & 55 & 55 & $\mathbf{5 5}$ \\
A.M. & 73 & 70 & 68 & 66 & 64 & $\mathbf{6 2}$ \\
\hline
\end{tabular}

Table 4. Initial and final tests for the body mass index of male gymnasts

\begin{tabular}{lcc}
\hline \multicolumn{3}{c}{ Body mass index - Male gymnasts } \\
\multicolumn{1}{c}{ Name } & IT & FT \\
\hline P.I. & 28.1 & 24.2 \\
M.A. & 23.8 & 21.9 \\
A.M. & 24.8 & 21.6 \\
S.I. & 23.4 & 22.8 \\
\hline
\end{tabular}

Noteworthy is the effort of gymnast A.M. to lose $11 \mathrm{~kg}$ and return to optimum weight for the competition (Table 5), but unfortunately he was no longer part of the gymnastics delegation.

Table 5. BMI grid

\begin{tabular}{cccccc}
\hline Age & Underweight & Normal Weight & Overweight & Obesity & Severe Obesity \\
\hline $18-24$ & $<19$ & $19-24$ & $24-29$ & $29-39$ & $>39$ \\
$25-34$ & $<20$ & $20-25$ & $25-30$ & $30-40$ & $>40$ \\
$35-44$ & $<21$ & $21-26$ & $26-31$ & $31-41$ & $>41$ \\
\hline
\end{tabular}


Some very important quantitative reference values for the workouts of both male and female gymnasts Levels I and II are shown in Tables 6-10.

Table 6. Quantitative training values for gymnasts

\begin{tabular}{lc}
\hline \multicolumn{1}{c}{ Workouts } & Hours \\
\hline Number of months & $\mathbf{7}$ \\
Number of weeks & $\mathbf{2 6}$ \\
Number of rest weeks & $\mathbf{5}$ \\
Number of workouts & $\mathbf{1 2 0}$ \\
Number of hours/workout & $\mathbf{2 ~ h ~ 1 / 2}$ \\
Total number of training hours & $\mathbf{2 7 9}$ \\
\hline
\end{tabular}

Table 7. Quantitative training values for female gymnasts - Level I

\begin{tabular}{lccccc}
\hline \multicolumn{1}{c}{ Female gymnasts - Level I } & \multicolumn{2}{c}{ Total } \\
\cline { 1 - 5 } \multicolumn{1}{c}{ Apparatus } & Vault & Uneven Bars & Beam & Floor & \\
\hline No. of elements/exercise & 2 & 6 & 10 & 10 & 28 \\
No. of full routines/training & 40 & 15 & 15 & 10 & 80 \\
No. of full routines/week & 204 & 77 & 77 & 52 & 410 \\
No. of full routine/all workouts & 4040 & 1540 & 1540 & 1040 & 8160 \\
\hline
\end{tabular}

Table 8. Quantitative training values for male gymnasts - Level I

\begin{tabular}{lcccccccc}
\hline \multicolumn{1}{c}{ Male gymnasts - Level I } & & Total \\
\cline { 1 - 7 } \multicolumn{1}{c}{ Apparatus } & Floor & $\begin{array}{c}\text { Pommel } \\
\text { Horse }\end{array}$ & Rings & Vault & $\begin{array}{c}\text { Parallel } \\
\text { Bars }\end{array}$ & $\begin{array}{c}\text { Horizontal } \\
\text { Bar }\end{array}$ & \\
\hline No. of elements/exercise & 10 & 10 & 8 & 2 & 12 & 8 & 50 \\
No. of full routines/training & 10 & 15 & 10 & 40 & 10 & 10 & 95 \\
No. of full routines/week & 52 & 75 & 52 & 204 & 52 & 52 & 487 \\
No. of full routine/all workouts & 1040 & 1500 & 1040 & 4040 & 1040 & 1040 & 9700 \\
\hline
\end{tabular}

Table 9. Quantitative training values for female gymnasts - Level II

\begin{tabular}{lccccc}
\hline \multicolumn{1}{c}{ Female gymnasts - Level II } & \multicolumn{2}{c}{ Total } \\
\cline { 1 - 5 } \multicolumn{1}{c}{ Apparatus } & Vault & Uneven Bars & Beam & Floor & \\
\hline No. of elements/exercise & 2 & 10 & 10 & 10 & 32 \\
No. of full routines/training & 40 & 6 & 10 & 10 & 66 \\
No. of full routines/week & 204 & 32 & 52 & 52 & 340 \\
No. of full routine/all workouts & 4040 & 640 & 1040 & 1040 & 6760 \\
\hline
\end{tabular}

Table 10. Quantitative training values for male gymnasts - Level II

\begin{tabular}{lcccccccc}
\hline \multicolumn{1}{c}{ Male gymnasts - Level II } & \multicolumn{2}{c}{ Total } \\
\cline { 1 - 7 } \multicolumn{1}{c}{ Apparatus } & Floor & $\begin{array}{c}\text { Pommel } \\
\text { Horse }\end{array}$ & Rings & Vault & $\begin{array}{c}\text { Parallel } \\
\text { Bars }\end{array}$ & $\begin{array}{c}\text { Horizontal } \\
\text { Bar }\end{array}$ & \\
\cline { 1 - 7 } No. of elements/exercise & 10 & 10 & 10 & 2 & 10 & 8 & 50 \\
No. of full routines/training & 10 & 15 & 6 & 40 & 10 & 5 & 86 \\
No. of full routines/week & 52 & 77 & 32 & 204 & 52 & 27 & 444 \\
No. of full routine/all workouts & 1040 & 1540 & 640 & 4040 & 1040 & 540 & 8840 \\
\hline
\end{tabular}


Table 11. Ranks of female gymnasts at the 2019 SO WSG competition

\begin{tabular}{lcccccc}
\hline & \multicolumn{1}{c}{ Female gymnasts - Levels I and II } & \multirow{2}{*}{ IC } \\
\cline { 1 - 4 } & Apparatus & Vault & Uneven Bars & Beam & Floor & \\
\hline I. D. & 3 & 3 & 3 & 1 & 2 \\
I. A.M. & 6 & 6 & 4 & 4 & 3 \\
B. M. & 7 & 5 & 4 & 2 & 7 \\
M. M. & 2 & 2 & 1 & 1 & 1 \\
\hline
\end{tabular}

Table 12. Ranks of male gymnasts at the 2019 SO WSG competition

\begin{tabular}{lccccccc}
\hline \multicolumn{7}{c}{ Male gymnasts - Levels I and II } & IC \\
\cline { 1 - 6 } \multicolumn{1}{c}{ Apparatus } & Floor & Pommel Horse & Rings & Vault & Parallel Bars & Horizontal Bar & \\
\hline A.M. & $\mathrm{X}$ & $\mathrm{X}$ & $\mathrm{X}$ & $\mathrm{X}$ & $\mathrm{X}$ & $\mathrm{X}$ & $\mathrm{X}$ \\
M.A. & 3 & 1 & 1 & 5 & 6 & 2 & 3 \\
S.I. & 3 & 3 & 4 & 1 & 3 & 3 & 3 \\
P.I. & 1 & 2 & 1 & 2 & 1 & 1 & 1 \\
\hline
\end{tabular}

Over 140 qualitative reference elements relating to the execution of elements, parts of exercise, full exercises, competitive performance, qualifications and finals, levels I and II, boys and girls, ensure the analytical, temporal and structural process of training and the monitoring of technical gains. Of these, the most significant data provided by the experiment are the average values obtained by gymnasts in the initial and final tests regarding the execution of full exercises in the 10 tests. The significant progress found was statistically confirmed for each test and level by the average score and Student's t-test (Table 13 and Figure 1).

Table 13. Average values obtained by gymnasts in the initial and final tests for full exercises on the 6 apparatus

\begin{tabular}{|c|c|c|c|c|c|c|c|c|c|c|c|c|c|}
\hline \multicolumn{2}{|c|}{ Apparatus } & \multicolumn{2}{|c|}{ Floor } & \multicolumn{2}{|c|}{ Pommel Horse } & \multicolumn{2}{|c|}{ Rings } & \multicolumn{2}{|c|}{ Vault } & \multicolumn{2}{|c|}{ Parallel Bars } & \multicolumn{2}{|c|}{ Horizontal Bar } \\
\hline Level & Gymnast & IT & FT & IT & FT & IT & FT & IT & FT & IT & FT & IT & FT \\
\hline I & A.M. & 7.3 & 9.4 & 8.4 & 9.2 & 8 & 9.5 & 8.3 & 9.2 & 7.1 & 9.4 & 7.2 & 9 \\
\hline I & M.A. & 7.86 & 9.16 & 6.41 & 8.92 & 8.04 & 9.35 & 8.17 & 9.19 & 7.46 & 9.06 & 7.78 & 9.22 \\
\hline II & S.I. & 7.3 & 8.9 & 5.4 & 8.7 & 8.1 & 9.2 & 8.1 & 9.2 & 7.1 & 8.8 & 7.2 & 9 \\
\hline II & P.I. & 8.7 & 9.5 & 6.4 & 9 & 8.8 & 9.7 & 8.7 & 9.3 & 8.1 & 9.1 & 8.2 & 9.5 \\
\hline
\end{tabular}

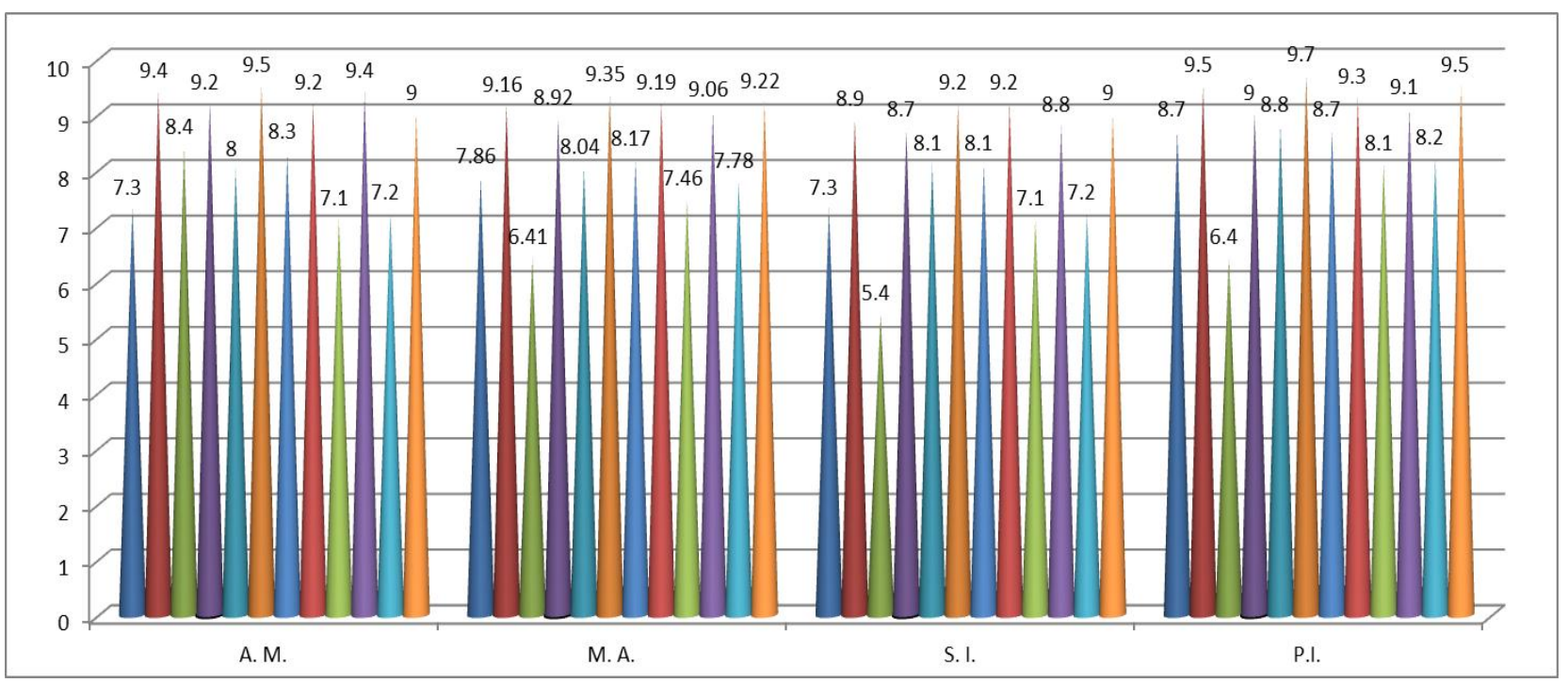


Figure 1. Average values obtained by gymnasts in the initial and final tests for full exercises on the 6 apparatus

\section{Conclusion}

Through this study, we managed to identify the most important features specific to the training of gymnasts with Down syndrome and also to select significant qualitative and quantitative benchmarks and reference points on how to guide, objectify and standardise the special preparation for major competitions.

For the achievement of outstanding performance, the training must follow the scheduled operational elements and particularly the algorithmic programmes of learning the elements, combinations and exercises. It must be continuously monitored through quantitative and qualitative parameters, and regularly assessed through verification competitions. It must be strategically related to reference items; we presented 30 , but there are many other analyses.

The amount of work to be done is very important. Hundreds and thousands of repetitions (see Tables 6 and 7) helped our athletes overcome all kinds of difficulties (such as a week without precompetitive training, superior emotional control, constancy and safety in execution) in both qualifying and finals competitions.

But be careful, the training must be sustained by the continuous respect of the positive relationship between volume-quality, safety, constancy. Equally important is to constantly monitor the training process, to model and stage the learning, to conduct verification competitions.

Based on the data and feedback, the content and means of the training for competition must be individually adjusted. In fact, the entire training programme should be subordinated to each gymnast's particularities for the level addressed.

All the values presented become reference points in the preparation of gymnasts with Down syndrome for high-level competitions. They will support worldwide specialists involved in the training of gymnasts with Down syndrome to achieve better quality and higher performance, as well as special gymnasts, who will have new opportunities to demonstrate their potential and aspirations for normality.

\section{References}

Ayers, S., \& Sariscsany, M. (2011). Physical education for lifelong fitness: The physical best teacher's guide (3 ${ }^{\text {rd }}$ ed.). Champaign, IL: Human Kinetics.

Damian, M., Popa, C., \& Popa, C. (2015). Bosu exercises for improvement of balance to children with Down syndrome. Gymnasium: Scientific Journal of Education, Sports \& Health, 16(Suppl.), 151-166. Retrieved from http://www.gymnasium.ub.ro/index.php/journal/article/view/127

Kerstiens, R. L., \& Green, M. (2015). Exercise in individuals with Down syndrome: A brief review. International Journal of Exercise Science, 8(2), 192-201. Retrieved from https://digitalcommons.wku.edu/ijes/vol8/iss2/10/

Monsen, L. (2019). United Arab Emirates hosts the 2019 Special Olympics World Games. Retrieved from https://share.america.gov/abu-dhabi-hosts-2019-special-olympics-world-games/

Popescu, G., Dina, L., Stroescu, S., \& Dina, G. (2013). Gymnastics motor learning particularities in Down syndrome children. Procedia - Social and Behavioral Sciences, 93, 2109-2113. https://doi.org/10.1016/j.sbspro.2013.10.174

SO Romania. (2019). 8 Sports/ 35 Athletes. Retrieved from https://www.abudhabi2019.org/delegation/soromania\# 